\title{
Possible Case of Novel Spotted Fever Group Rickettsiosis in Traveler Returning to Japan from India
}

\section{Ichiro Takajo, ${ }^{1}$ Tsuyoshi Sekizuka, Hiromi Fujita, Ayako Kawano, Takeshi Kawaguchi, Motohiro Matsuda, Kazuyoshi Kubo, Shunichi Miyauchi, Kunihiko Umekita, Yasuhiro Nagatomo, Makoto Kuroda, Tomohiko Takasaki, Akihiko Okayama, Shuji Ando'1}

A 60-year-old woman experienced fever, headache, rash, and altered vision after returning to Japan from India. Testing detected elevated antibody titers to spotted fever group rickettsia; PCR on blood yielded positive results for the rickettsial outer membrane protein A gene. We isolated a unique rickettsial agent and performed a full-genome analysis.

$\mathrm{V}$ arious types of spotted fever group (SFG) rickettsioses have been reported worldwide (1). Common symptoms include fever, headache, intense myalgia, and skin rash (2). Even though cases of spotted fever have occurred in Japan, physicians often face difficulty diagnosing the disease in febrile patients because of the unusual or unfamiliar symptoms associated with SFG rickettsia.

In January 2011, a 60-year-old woman from Japan who was undergoing treatment for diabetes mellitus stayed in a suburban area of Bangalore in South India for 1 week. She went camping and did other outdoor activities. She recalled that there were many opportunities to be bitten by insects; however, she could not specify the types of insects to which she may have been exposed. On her first day back in Japan (day 1), she experienced general malaise and loss of appetite. A fever $\left(38^{\circ} \mathrm{C}\right)$ and skin rash appeared on day 7. She consulted a local clinic and underwent evaluation. Blood test results indicated thrombocytopenia $(91 \times$ $10^{9}$ thrombocytes/L), liver dysfunction (elevated aspartate aminotransferase [92 U/L] and alanine transaminase [97 $\mathrm{U} / \mathrm{L}]$ ), and elevated C-reactive protein levels $(206 \mathrm{mg} / \mathrm{L})$. Splenomegaly was evident on abdominal sonography. On day 12 , the woman was admitted to Miyazaki University

Author affiliations: University of Miyazaki, Miyazaki, Japan

(I. Takajo, A. Kawano, T. Kawaguchi, M. Matsuda, K. Kubo,

S. Miyauchi, K. Umekita, Y. Nagatomo, A. Okayama); National

Institute of Infectious Diseases, Tokyo, Japan (T. Sekizuka,

M. Kuroda, T. Takasaki, S. Ando); Mahara Institute of Medical

Acarology, Tokushima, Japan (H. Fujita)

DOI: http://dx.doi.org/10.3201/eid2206.151985
Hospital (Miyazaki, Japan) with a high fever $\left(39.4^{\circ} \mathrm{C}\right)$, headache, altered vision with eye floaters, and rash (Figure 1, panel A). Proteinuria, glycosuria, thrombocytopenia $\left(92 \times 10^{9}\right.$ thrombocytes/L), liver dysfunction (elevated aspartate aminotransferase $[95 \mathrm{U} / \mathrm{L}]$ and alanine aminotransferase $[93 \mathrm{U} / \mathrm{L}]$ ), and hyponatremia (sodium $127 \mathrm{mmol} / \mathrm{L}$ ) were observed, and levels of serum procalcitonin (1.9 ng/ $\mathrm{mL})$ and C-reactive protein $(198 \mathrm{mg} / \mathrm{L})$ were elevated. Her diabetes was poorly controlled (glucose $40.5 \mathrm{mmol} / \mathrm{L}$ ).

A skin biopsy of the rash was performed on day 13; infiltration of inflammatory cells (mainly lymphocytes) around the capillaries, associated with hemorrhagic changes, was observed (Figure 1, panel B). No eschars were detected. Given the patient's travel history to India and her signs and symptoms, we considered acute infectious diseases such as dengue fever, chikungunya fever, and typhoid fever. Ceftriaxone $(2 \mathrm{~g} / \mathrm{d})$ and levofloxacin $(500 \mathrm{mg} / \mathrm{d})$ were administered. Subsequently, the fever subsided, and platelet count, liver function, and C-reactive protein level returned to normal. However, laboratory findings ruled out the principal tropical infectious diseases. Blood smear specimens tested negative for malaria parasites. Blood culture tested negative for bacteria, thus ruling out typhoid fever. Dengue and chikungunya virus infections were ruled out on the basis of serologic tests and antigen detection. Although the rash, bilateral lower limb edema, and visual alterations with eye floaters persisted, the patient was discharged on day 27.

A serum sample revealed apparent positivity to Rickettsia conorii Malish 7 and $R$. japonica $\mathrm{YH}$ on day 19 (Table). Therefore, SFG rickettsia was strongly suspected. By day 40, the patient's rash and edema persisted, and her visual alterations had become more severe. Because persistent SFG rickettsiosis was suspected, additional oral treatment with minocycline $(100 \mathrm{mg} / \mathrm{d})$ was administered for 14 days. By day 48, the remaining symptoms resolved.

PCR test results for the acute-phase whole blood sample collected on day 13 were positive for the outer membrane protein A (ompA) gene for SFG rickettsia $(3,4)$. Direct nucleotide sequencing of PCR products (GenBank accession no. LC089865) yielded a profile different from any known Rickettsia spp. but identical to that of Rickettsia sp. CMCMICRO 1-4 (GenBank accession nos. HM587248.1-HM587251.1) (5). We successfully isolated an SFG rickettsial agent, designated as strain Tenjiku01,

${ }^{1}$ These authors contributed equally to this article. 

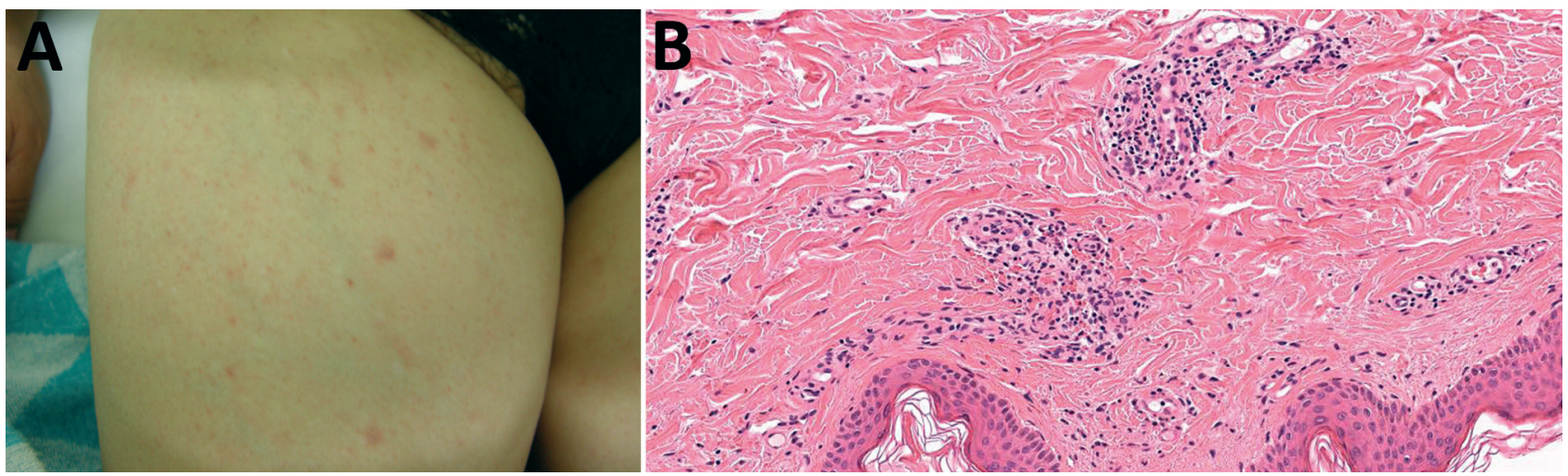

Figure 1. Rash and skin biopsy of a 60-year-old female traveler who had returned to Japan from India, January 2011. A) Rash on the inner side of the right thigh. B) Skin biopsy showing infiltration of inflammatory cells (mainly lymphocytes) around the capillaries, associated with hemorrhagic changes (hematoxylin-eosin staining, original magnification $\times 400$ ).

from acute-phase whole blood (collected on day 13) using the shell vial method with L929 cells. The ompA sequence of the isolate was identical to that of the clinical sample (online Technical Appendix 1 Figure, http://wwwnc.cdc. gov/EID/article/20/6/15-1985-Techapp1.pdf). In addition to partial sequencing of PCR products (GenBank accession nos.: $16 S$ rRNA, LC089861; 17K-Da, LC089862; geneD, LC089863; gltA, LC089864), we performed wholegenome analysis. The partial sequence of ompA from our PCR products was $100 \%$ similar to the CMCMICRO ompA sequence (5) and $98.4 \%$ similar to the Candidatus R. kellyi ompA sequence (6). Other genes with high sequence homology were as follows: gltA with uncultured Rickettsia sp. LIC4275 (99.7\% homology, accession no. KT153042); rrs with $R$. slovaca 13-B (99.7\%, NR_074462); sca4 (geneD) with $R$. slovaca $13-\mathrm{B}(98.4 \%, \overline{\mathrm{CP}} 002428)$; and $17 \mathrm{~K}-\mathrm{Da}$ gene with $R$. honei RB (99.5\%, AF060704). The draft genome sequence $(\approx 1.3 \mathrm{Mb}, 32$ contigs $)$ was obtained with next-generation sequencing (accession nos. BCMR01000001-BCMR01000032). The outer membrane protein B sequence, which was extracted from the contig by next-generation sequencing, was $96.98 \%$ similar to that of $R$. slovaca D-CWPP (accession no. CP003375). Results of pan-genome analysis suggested that 586 core genes were shared among 34 Rickettsia spp. genomes, and the gene components of Tenjiku01 were highly similar to those of the SFG group (data not shown). A maximum-likelihood phylogenetic tree of concatenated amino acid sequence alignments of the core genes, constructed by using RAxML software version 8.2.0 (http://sco.h-its.org/exelixis/ web/software/raxml), indicated that Tenjiku01 belongs to the SFG group and is closely related to $R$. honei RB (Figure 2). Moreover, blastp matrix analysis (http://blast.ncbi. nlm.nih.gov) of 73 Rickettsia spp. indicated that 315 core genes of Tenjiku01 showed $98.21 \%-98.95 \%$ homology to those of $R$. japonica, $R$. argasii, $R$. heilongjiangensis, $R$. honei, $R$. parkeri, $R$. sibirica, $R$. africae, and $R$. conorii (online Technical Appendix 2, http://wwwnc.cdc.gov/EID/ article/22/6/15-1985-Techapp2.xlsx).

At first, this patient's infection partially responded to levofloxacin and ceftriaxone therapy. The efficacy of levofloxacin in treating Rickettsia spp. has been described previously (7). Therefore, levofloxacin, not ceftriaxone, probably was effective in this patient. After detection of SFG rickettsia antibodies, we performed PCR analysis, which resulted in the final diagnosis; the patient's remaining symptoms were then successfully treated with minocycline.

Recently, many types of SFG rickettsia, except $R$. japonica, have occurred in Japan $(4,8)$. An even greater variety of rickettsioses has been reported worldwide $(1,9,10)$, and the incidence of imported rickettsioses has increased in Japan (11-13).

In our patient, antibody titers to $R$. conorii and $R$. japonica were elevated on day 19 , and the titers to $R$. conorii were higher than those against $R$. japonica. Therefore, we

\begin{tabular}{|c|c|c|c|}
\hline Species & Day 13 & Day 19 & Day 40 \\
\hline Rickettsia japonica (YH) & - & $1: 640$ & $1: 640$ \\
\hline R. conorii (Malish7) & - & $1: 2,560$ & $1: 5,120$ \\
\hline R. typhi (Wilmington) & - & $1: 40$ & $1: 160$ \\
\hline R. prowazekii (Brainl) & - & $1: 40$ & $1: 80$ \\
\hline Orientia tsutsugamushi (Karp) & - & - & - \\
\hline O. tsutsugamushi (Gilliam) & - & - & - \\
\hline
\end{tabular}

*-, negative. 


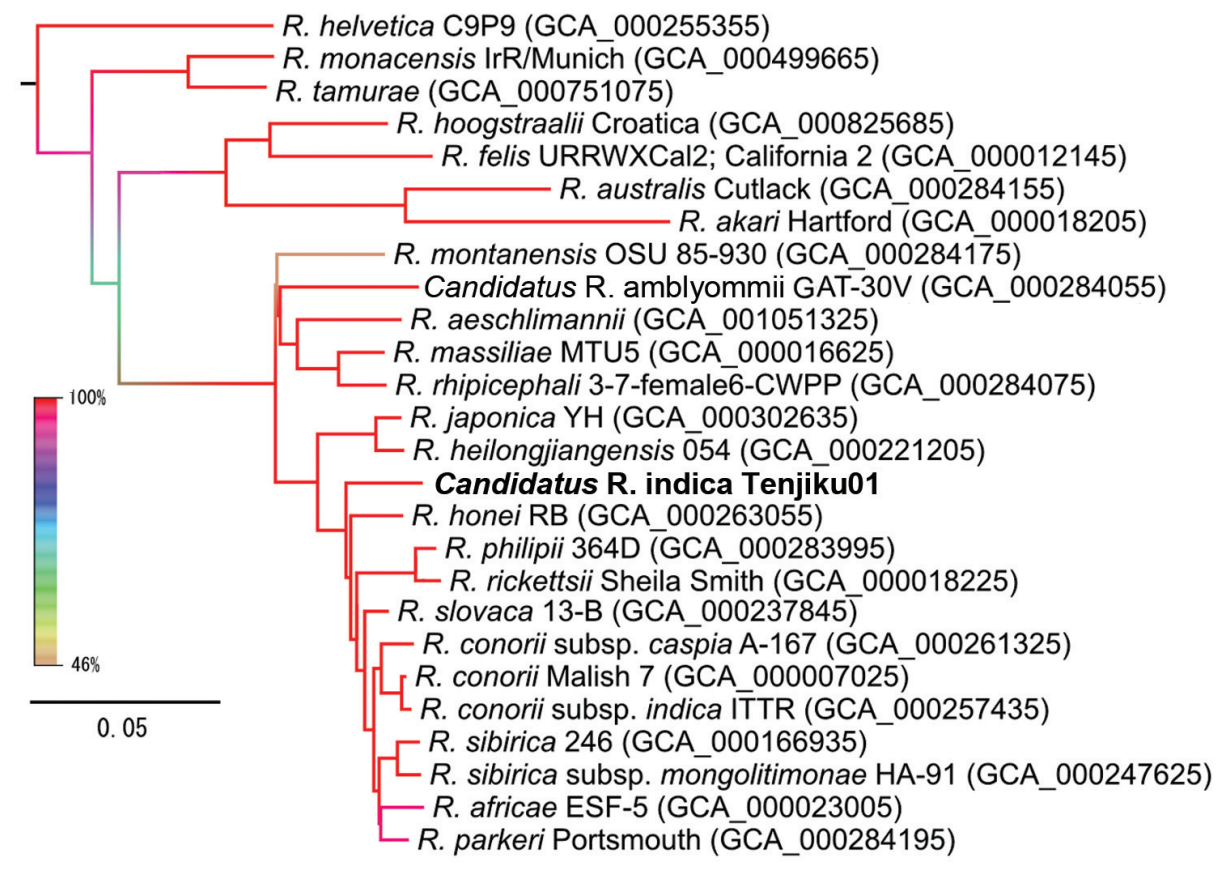

Figure 2. Maximum-

likelihood phylogenetic tree of concatenated core genes in 26 Rickettsia spp. strains constructed by using RAxML software version 8.2.0 (http://sco.h-its.org/exelixis/web/ software/raxml) with 1,000-fold bootstrapping. Boldface indicates isolate from this study. The color of each branch represents the bootstrapping value. GenBank assembly accession numbers are given in parentheses. Scale bar indicates amino acid changes per position. suspected that the causative pathogen was closely related to $R$. conorii.

The ompA PCR products amplified from clinical samples were identical to the sequences of Rickettsia sp. CMCMICRO registered in India. Prakash et al. tested skin biopsy samples for SFG rickettsial genes and concluded that novel species of SFG rickettsia (CMCMICRO1-8) were in their area (5). However, isolation of SFG rickettsia has not been reported thus far. Moreover, data on other genes, such as $17 K D a$, gltA, and gene D, are lacking (5). Little sequence homology was observed between the omp $A$ sequence in our case and that of Candidatus R. kellyi (6), which is considered to be the most closely related to Rickettsia sp. CMCMICRO, according to Fournier's criteria (14). In our case, we successfully isolated SFG rickettsia, Rickettsia sp. strain Tenjiku01, from the clinical sample. Comparative genomics suggested that Tenjiku01 could be a novel species because the phylogenetic distance between Tenjiku01 and $R$. honei RB was longer than that between Tenjiku01 and other similar species.

\section{Conclusions}

We successfully diagnosed imported SFG rickettsiosis in a traveler returning to Japan from India on the basis of serology and molecular laboratory techniques. If a patient reports a recent history of travel abroad, physicians should consider SFG rickettsia in the differential diagnosis. Our analysis will help elucidate a variety of rickettsial pathogenicities and biologic characteristics reported worldwide. On the basis of our findings, we propose this isolate as a novel species, Candidatus Rickettsia indica.
This work was supported by the grants for Research on Emerging and Re-Emerging Infectious Diseases (H24 Shinko-Ippan-008 and H25 Shinko-Ippan-015) from the Ministry of Health, Labor, and Welfare, Japan.

Dr. Takajo is a clinician specializing in infectious diseases and infection control at the Department of Rheumatology, Infectious Disease, and Laboratory Medicine, Faculty of Medicine, University of Miyazaki, Japan. His primary research interest is the nosocomial infection control.

\section{References}

1. Parola P, Paddock CD, Socolovschi C, Labruna MB, Mediannikov O, Kernif T, et al. Update on tick-borne rickettsioses around the world: a geographic approach. Clin Microbiol Rev. 2013;26:657-702. http://dx.doi.org/10.1128/CMR.00032-13

2. Walker DH. Rickettsiae and rickettsial infections: the current state of knowledge. Clin Infect Dis. 2007;45(Suppl 1):S39-44. http://dx.doi.org/10.1086/518145

3. Regnery RL, Spruill CL, Plikaytis BD. Genotypic identification of rickettsiae and estimation of intraspecies sequence divergence for portions of two rickettsial genes. J Bacteriol. 1991;173:1576-89.

4. Ando S, Kurosawa M, Sakata A, Fujita H, Sakai K, Sekine M, et al. Human Rickettsia heilongjiangensis infection, Japan. Emerg Infect Dis. 2010;16:1306-8. http://dx.doi.org/10.3201/eid1608.100049

5. Prakash JA, Sohan Lal T, Rosemol V, Verghese VP, Pulimood SA, Reller M, et al. Molecular detection and analysis of spotted fever group Rickettsia in patients with fever and rash at a tertiary care centre in Tamil Nadu, India. Pathog Glob Health. 2012;106:40-5. http://dx.doi.org/10.1179/2047773212Y.0000000001

6. Rolain JM, Mathai E, Lepidi H, Somashekar HR, Mathew LG, Prakash JAJ, et al. "Candidatus Rickettsia kellyi," India. Emerg Infect Dis. 2006;12:483-5. http://dx.doi.org/10.3201/ eid1203.050853

7. Maurin M, Raoult D. Bacteriostatic and bactericidal activity of levofloxacin against Rickettsia rickettsii, Rickettsia conorii, 'Israeli 
spotted fever group rickettsia' and Coxiella burnetii. J Antimicrob Chemother. 1997;39:725-30. http://dx.doi.org/10.1093/jac/39.6.725

8. Imaoka K, Kaneko S, Tabara K, Kusatake K, Morita E. The first human case of Rickettsia tamurae infection in Japan. Case Rep Dermatol. 2011;3:68-73. http://dx.doi.org/10.1159/000326941

9. Parola P, Raoult D. Ticks and tickborne bacterial diseases in humans: an emerging infectious threat. Clin Infect Dis. 2001; 32:897-928. http://dx.doi.org/10.1086/319347

10. Kelly DJ, Fuerst PA, Ching WM, Richards AL. Scrub typhus: the geographic distribution of phenotypic and genotypic variants of Orientia tsutsugamushi. Clin Infect Dis. 2009;48(Suppl 3):S20330. http://dx.doi.org/10.1086/596576

11. Freedman DO, Weld LH, Kozarsky PE, Fisk T, Robins R, von Sonnenburg F, et al. Spectrum of disease and relation to place of exposure among ill returned travelers. N Engl J Med. 2006;354:119-30. http://dx.doi.org/10.1056/NEJMoa051331

12. Fujisawa T, Kadosaka T, Fujita H, Ando S, Takano A, Ogasawara Y, et al. Rickettsia africae infection in a Japanese traveller with many tick bites. Acta Derm Venereol. 2012;92:443-4.

http://dx.doi.org/10.2340/00015555-1313

13. Yoshikawa H, Kimura M, Ogawa M, Rolain JM, Raoult D. Laboratory-confirmed Mediterranean spotted fever in a Japanese traveler to Kenya. Am J Trop Med Hyg. 2005;73:1086-9.

14. Fournier PE, Raoult D. Current knowledge on phylogeny and taxonomy of Rickettsia spp. Ann N Y Acad Sci. 2009;1166:1-11. http://dx.doi.org/10.1111/j.1749-6632.2009.04528.x_

Address for correspondence: Ichiro Takajo, Department of Rheumatology, Infectious Disease, and Laboratory Medicine, Faculty of Medicine, University of Miyazaki, 5200 Kihara, Miyazaki 889-1692, Japan; email: ichiro_takajo@med.miyazaki-u.ac.jp; or Shuji Ando, Department of Virology I, National Institute of Infectious Diseases, 1-23-1 Toyama, Shinjuku-ku, Tokyo 162-8640, Japan; email: shuando@nih.go.jp

\section{August 2015: Surveillance Including:}

- Escherichia coli O157 Outbreaks in the United States, 2003-2012

- Underrecognition of Dengue during 2013 Epidemic in Luanda, Angola

- Health Care-Associated Infection Outbreak Investigations in Outpatient Settings, Los Angeles County, California, USA, 2000-2012

- Differentiation of Acute Q Fever from Other Infections in Patients Presenting to Hospitals, the Netherlands

- Community-Based Outbreak of Neisseria meningitidis Serogroup C Infection in Men who Have Sex with Men, New York City, New York, USA, 2010-2013

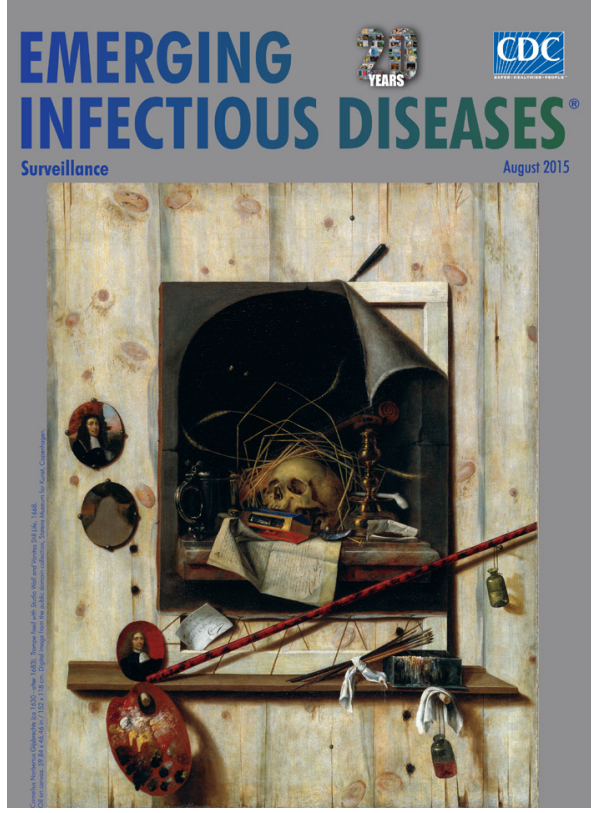

- Risk for Mycobacterial Disease among Patients with Rheumatoid Arthritis, Taiwan, 2001-2011

- Phylogeography of Influenza A(H3N2) Virus in Peru, 2010-2012

- Susceptibility of Carrion Crows to Experimental Infection with Lineage 1 and 2 West Nile Viruses

- Response Strategies against Meningitis Epidemics after Elimination of Serogroup A Meningococci, Niger

- Influenza A Viruses of Human Origin in Swine, Brazil

- Prevalence of Hepatitis E Virus Infection in Pigs at the Time of Slaughter, United Kingdom, 2013

- Development of Framework for Assessing Influenza Virus Pandemic Risk

http://wwwnc.cdc.gov/eid/articles/issue/21/08/table-of-contents 